\title{
Orofacial Symptoms Related to Temporomandibular Joint Arthritis in Juvenile Idiopathic Arthritis: Smallest Detectable Difference in Self-reported Pain Intensity
}

\author{
PETER STOUSTRUP, KASPER D. KRISTENSEN, CARLALBERTA VERNA, ANNELISE KÜSELER, \\ TROELS HERLIN, and THOMAS K. PEDERSEN
}

\begin{abstract}
Objective. Temporomandibular joint (TMJ) inflammation in patients with juvenile idiopathic arthritis (JIA) may lead to mandibular growth disturbances and interfere with optimal joint and muscle function. Orofacial symptoms are common clinical findings in relation to TMJ arthritis in adolescence. Knowledge about their clinical manifestation is important for TMJ arthritis diagnosis, treatment choice, and outcome evaluation. The aim of our prospective observational study was to evaluate and describe the frequency, the main complaints, and the localization of TMJ arthritisrelated orofacial symptoms. The smallest detectable differences (SDD) for minimal, average, and maximal pain were estimated.

Methods. Thirty-three patients with JIA and arthritis-related orofacial symptoms in relation to 55 affected TMJ were included in our questionnaire study (mean age $14.11 \mathrm{yrs}$ ). Calculation of the SDD was based on a duplicate assessment $45 \mathrm{~min}$ after the first questionnaire was completed.

Results. The majority of the patients had common orofacial symptoms during mastication and maximal mouth opening procedures. Persistent orofacial symptoms were rare. The TMJ area in combination with the masseter muscle region was the orofacial region where symptoms were most common. The SDD for minimal, average, and maximal pain were between 10 and $14 \mathrm{~mm}$ on a visual analog scale.

Conclusion. Our study offers new knowledge about TMJ arthritis-related orofacial symptoms that may aid diagnosis and clinical decision-making. We suggest that TMJ arthritis-related orofacial symptoms could be understood as products of the primary TMJ inflammation in combination with secondary myogenic and functional issues. (J Rheumatol First Release Oct 1 2012; doi:10.3899/ jrheum.120437)
\end{abstract}

Key Indexing Terms:

TEMPOROMANDIBULAR JOINT

OROFACIAL PAIN

JUVENILE ARTHRITIS

TEMPOROMANDIBULAR DISORDER RELIABILITY

Juvenile idiopathic arthritis (JIA) is an arthritic condition of unknown etiology. It is defined by an onset of arthritis before the age of 16 years, persisting for $>6$ weeks ${ }^{1}$. The prevalence of temporomandibular joint (TMJ) arthritis in JIA reaches $30 \%-87 \%$ depending on diagnostic criteria and methodology $2,3,4,5,6,7$. TMJ arthritis may interfere with optimal joint function $6,8,9$. TMJ pain and functional impairment have limited sensitivity as predictors of TMJ inflammation and clinical signs of inflammation may not necessarily be present in patients with TMJ arthritis $4,8,10$. The presence of symptoms and functional impairment of the

From the Department of Orthodontics and the Department of Pediatrics, Aarhus University Hospital, Aarhus, Denmark.

P. Stoustrup, DDS, PhD; K.D. Kristensen, DDS, PhD; C. Verna, DDS, PhD; A. Küseler, DDS, PhD, Department of Orthodontics, Aarhus University; T. Herlin, MD, Professor, Department of Pediatrics, Aarhus University Hospital; T.K. Pedersen, DDS, PhD, Associate Professor, Department of Orthodontics, Aarhus University.

Address correspondence to Dr. P. Stoustrup, Department of Orthodontics, Aarhus University, Vennelyst Blvd. 9-11, 8000 Aarhus, Denmark.

E-mail:pstoustrup@odont.au.dk.

Accepted for publication August 8, 2012.
TMJ and its surrounding soft tissue is seen in $26 \%-74 \%$ of JIA patients with TMJ arthritis, and there is a positive correlation between disease duration and craniofacial pain and dysfunction $^{8,11}$. The main complaints that patients with TMJ arthritis have reported are reduced maximal opening capacity, pain during mouth opening, tiredness of the jaws, and TMJ crepitation; however, subjective dysfunction symptoms are almost absent in patients below the age of 7 years $^{8,11,12}$.

Intervention studies of patients with JIA and TMJ arthritis have proposed that statistically significant differences between pretherapeutic and posttherapeutic orofacial pain intensity may reflect the clinical outcome of the intervention. However, differences between pretherapeutic and posttherapeutic outcomes may be influenced by the natural course of the disease, biological fluctuation in the pain intensity, and inconsistency of the patients' self-assessment of this variable ${ }^{13,14}$. We cannot equate a significant change in this outcome variable in a therapeutic study with clinical relevance until the reliability and reproducibility of the pain intensity variable have been duly accounted for. In clinical

Personal non-commercial use only. The Journal of Rheumatology Copyright @ ${ }^{2012}$. All rights reserved. 
decision-making as well as within research, it is therefore important to have detailed knowledge of the "natural variation" in pain intensity among test persons.

A commonly applied clinical pain intensity measurement tool is a nonverbal visual analog scale (VAS) of $100 \mathrm{~mm}$, where the left extreme represents "no pain" and the right extreme represents the "worst imaginable pain." In research, consistency within the measurement of this scale is often expressed in terms of correlation coefficients ${ }^{15}$. Nilsson, et al described good reliability and high validity of the self-reported pain associated with temporomandibular disorders in an adolescent nonrheumatic group of patients, based on agreement between 2 consecutive pain intensity assessments ${ }^{16}$. However, a correlation coefficient does not inform the clinician about the amount of change needed to overcome the inconsistency quantified by the standard error of the measurement (SEM). Calculation of the smallest detectable difference (SDD) has been proposed as an appropriate method to address the variation of a measurement/ assessment ${ }^{14}$. According to Kropmans, et al the SDD is the minimal amount of change that can be identified clinically between 2 consecutive observations ${ }^{14,17}$. It offers the clinician information on the least difference below which anything smaller cannot be reliably distinguished from random error in 2 consecutive pain intensity assessments expressed in the same unity as the VAS scale. Knowledge about the SDD therefore lies at the root of any assessment of the outcome of an intervention.

The aims of our prospective observational questionnaire study of consecutive patients with JIA and TMJ arthritis-related orofacial pain were (1) to describe the sensory-discriminative components of self-reported orofacial symptoms; and (2) to estimate the SDD for selfreported minimal, average, and maximal orofacial pain based on consecutive VAS scales.

\section{MATERIALS AND METHODS}

Thirty-five consecutive JIA patients with previous or current TMJ arthritis and orofacial pain were identified as potential candidates for study. They were all called for examination and treatment of their orofacial pain and/or mandibular growth disturbances at the Department of Orthodontics, Aarhus University, Denmark, between January and July 2011. Inclusion criteria were (1) a JIA diagnosis according to the criteria of the International League of Associations for Rheumatology ${ }^{1}$; (2) a diagnosis of TMJ arthritis-related orofacial pain based on regular standardized clinical examination and radiological evaluation; and (3) being over 10 years of age and cognitively capable of understanding the concept of the VAS.

Diagnosis of TMJ arthritis-related orofacial pain. The initial TMJ arthritis diagnosis was based on a standardized orthodontic clinical examination protocol containing 2 parts: the first, subjective part featured the patient's self-assessment of current TMJ status. The patient answered 14 questions addressing subjects such as pain level, TMJ function, chewing capability, etc. The second, objective part contained 57 clinically relevant measurements assessed by the examiner, e.g., maximal mouth opening capacity, symmetry during opening, capability of laterotrusion and protrusion, etc. The clinical examination was supplemented with a radiological evaluation with cone-beam computerized tomography. The initial TMJ arthritis diagnosis was based on an overall assessment of the clinical and radiological findings after the diagnosis of JIA was given. The diagnosis of TMJ arthritis-related orofacial pain was given when the orofacial symptoms were specifically ascribed to current/previous TMJ inflammation based on the findings in the clinical examination. The initial TMJ arthritis diagnosis was not dependent on the presence of orofacial symptoms. However, the presence of orofacial symptoms was a specific inclusion criterion for our study.

The self-assessment questionnaire. In addition to our standardized orthodontic clinical examination protocol, all included candidates were asked to fill out a questionnaire concerning (1) the duration of their orofacial pain within the last week; (2) their orofacial pain frequency within the last week; (3) the child's account of his/her own orofacial symptoms ("In which situations do you feel the pain?"); (4) the localization of the symptoms marked on a map illustrating the head and the neck; and (5) a description of their average, maximal, and minimal pain intensity based on 3 individual VAS scores. Preexamination pain intensity assessment (T1) was followed by a second pain intensity assessment (T2) 45 min after the first questionnaire had been filled out. Candidates were again asked to fill out an identical questionnaire concerning their VAS assessment of their average, maximal, and minimal pain intensity within the last week. The patients were not informed about the contents of the second questionnaire until they were asked to complete it.

The examination complied with current national regulations for clinical examinations in pediatric orthodontics, and the study procedures were in accord with the Declaration of Helsinki. The study was approved by the Danish Data Protection Agency and by the Danish Ministry of Health. All included patients with JIA and their parents gave informed consent.

Statistics. All variables were tested for normal distribution. Intrapatient bias was evaluated graphically: the first VAS score was plotted against the second VAS score to check for systematic time trends. Moreover, paired t-tests between the first and the second VAS score were conducted for minimal, average, and maximal orofacial pain using SAS 9.2, PROC MIXED (SAS Institute Inc.).

The model describing the data is a variance component model with 2 components, i.e., the variation between patients and the variation between the measurements. Because we were interested in comparing 2 measurements on the same patient, the random variation between patients was irrelevant. The variance of the measurement was estimated using analysis of variance, and the SEM was calculated as the square root of the estimate. With a significance level of $\alpha=0.05$, the SDD was estimated for each of the variables of interest ${ }^{17,18}$ :

$$
\mathrm{SDD}=1.96 \times(\sqrt{2}) \times \mathrm{SEM}
$$

\section{RESULTS}

Thirty-three of the preidentified 35 candidates were included in our study. In this group, arthritis-related orofacial symptoms were seen in relation to a total of 55 TMJ in 11 patients with unilateral TMJ arthritis and 22 patients with bilateral TMJ arthritis. The majority of included patients were girls (31/33). Two patients were excluded because their symptoms were ascribed to other temporomandibular dysfunctions and not assessed as a precise product of current/previous TMJ inflammation. In those 2 patients, the orofacial symptoms were ascribed to bruxism and anterior disc displacements. The cohort characteristics of the patients are presented in Table 1. None of the patients had received their initial TMJ arthritis diagnosis recently. All patients had received previous regular clinical and radiological TMJ arthritis examinations in accordance 
Table 1. Characteristics of study patients.

\begin{tabular}{lc}
\hline Characteristics & Patients, $\mathrm{n}=33$ \\
\hline Females & $31(94 \%)$ \\
Mean age, yrs (range) & $14.11(11-18.6)$ \\
Duration of symptoms, $\mathrm{n}^{*}$ & \\
$\quad<3$ mo & 10 \\
$>3$ mo, $<12$ mo & 5 \\
$>12$ mo & 10 \\
Does not remember & 8 \\
TMJ involvement, $\mathrm{n}$ & \\
Unilateral & 11 \\
Bilateral & 22 \\
JIA diagnosis, $\mathrm{n}$ & \\
Oligoarticular & 12 \\
Polyarticular & 16 \\
Systemic & 2 \\
Psoriatic & 1 \\
Undifferentiated** & 2 \\
Medication, $\mathrm{n}$ & \\
NSAID & 11 \\
Paracetamol & 2 \\
Sulfasalazine & 2 \\
Methotrexate & 15 \\
IACI*** & 1 \\
Systemic steroids & 0 \\
Biologics & 8 \\
No medication & 7 \\
\hline Only oracian & \\
\hline & \\
\hline &
\end{tabular}

* Only orofacial symptoms. $25 / 33$ patients were able to remember the duration of their orofacial symptoms. ** Subtype diagnosis was still unknown at the time of examination. *** Intraarticular TMJ steroid injection within the past 3 months. TMJ: temporomandibular joint; JIA: juvenile idiopathic arthritis; NSAID: nonsteroidal antiinflammatory drugs; IACI: intraarticular corticosteroid injections. with our standardized orthodontic clinical examination protocol.

Frequency and main complaints of orofacial pain. The frequency of orofacial pain is presented in Figure 1. Almost all patients experienced orofacial pain on a weekly basis. However, only 1 patient reported constant orofacial pain. The majority of patients experienced TMJ and masticatory muscle symptoms during mastication and maximal mouth opening procedures. The mastication of hard and tough food objects was reported to be particularly problematic. Moreover, TMJ morning stiffness was also a frequent complaint. The patients' accounts of their own orofacial symptoms are presented in Figure 2.

Pain localization. The relative pain localization of the self-reported symptoms and the distribution of pain sites are presented in Figure 3. The majority of the patients reported well defined pain localizations. The pain localizations most often involved were the TMJ and the masseter muscle regions. In the majority of the patients with unilateral/ bilateral TMJ arthritis-related orofacial symptoms, both these regions were involved. Self-reported pain solely from the TMJ region was seen only in a smaller proportion of the patients. Moreover, some of the patients did not localize the TMJ area as the origin site of pain. Only a few patients reported pain from the region of the temporal muscle. A minor proportion of the patients reported diffuse pain that involved a broad proportion of the orofacial area.

SDD of the self-reported pain. The majority of patients were familiar with the concept of the VAS at inclusion. Table 2 shows the intrapatient agreement between T1 and T2 VAS

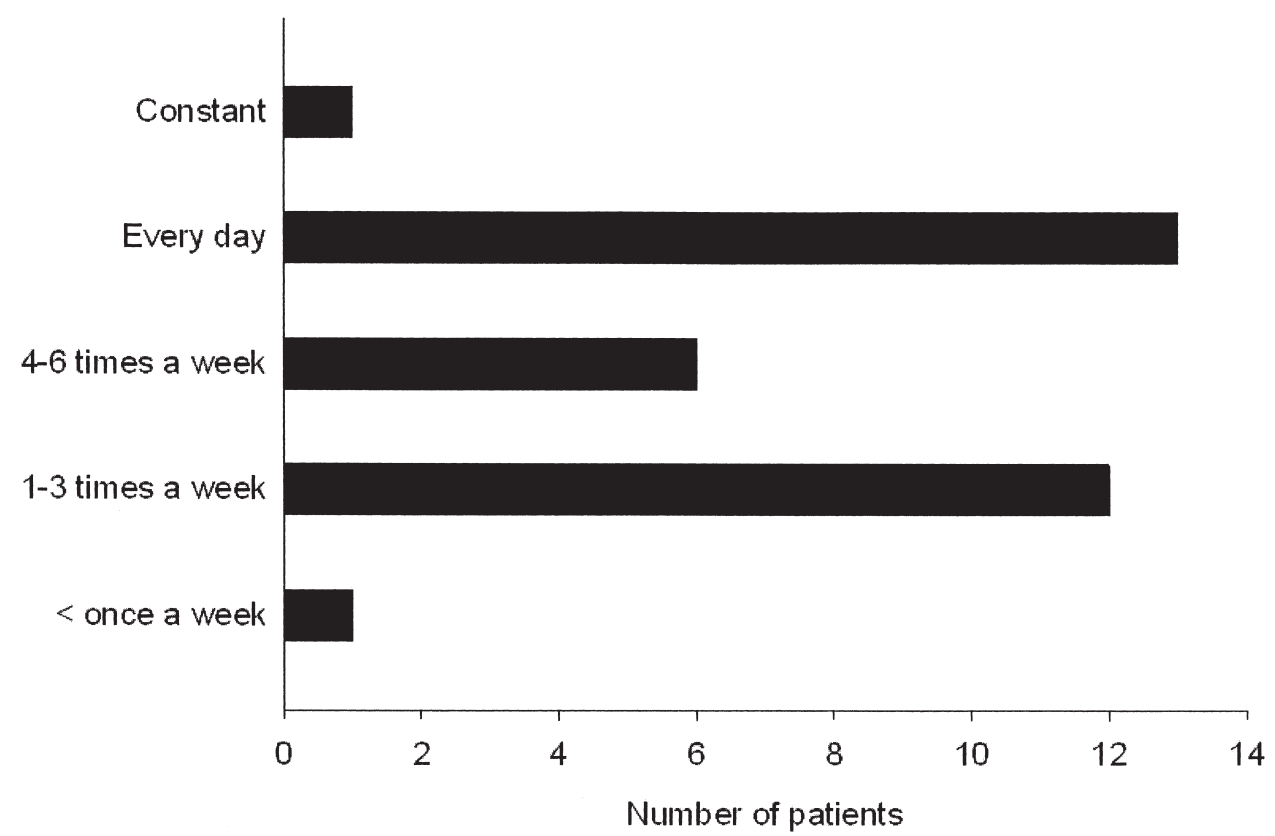

Figure 1. Orofacial pain frequency. "Constant" reflects constant pain during the day, while "every day" reflects episodes of orofacial symptoms. 


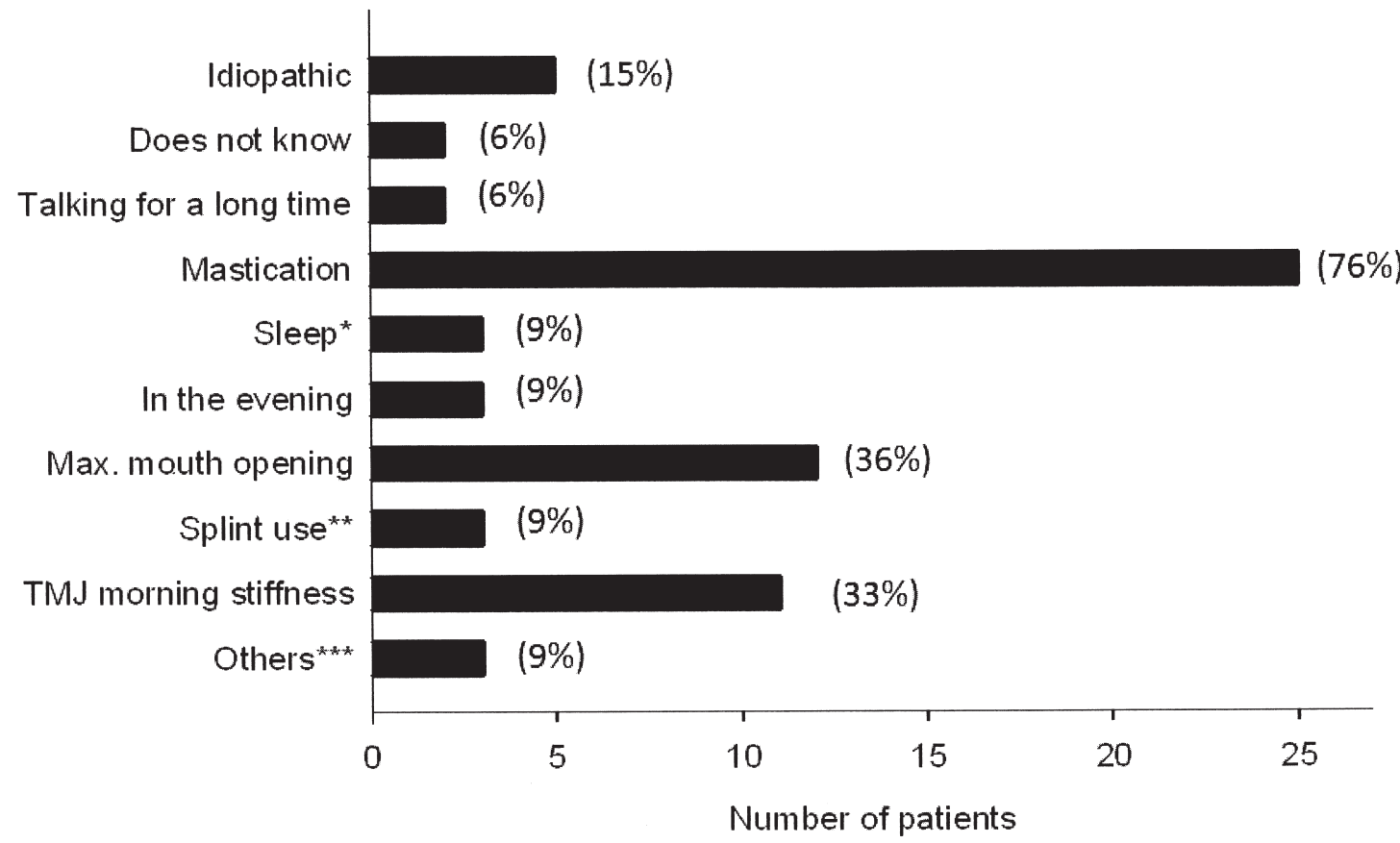

Figure 2. Self-reported main complaints. Unlimited numbers of complaints were allowed. *Complaints reflect a sleeping position that put strain on the affected joint unintentionally. **Two patients reported that not using the splint resulted in orofacial symptoms. One patient reported that constant splint use without breaks caused orofacial symptoms. ***Other complaints included (1 patient each): "when I am cold," "tiredness/fatigue," and "after having rested the mouth for a long time."

a

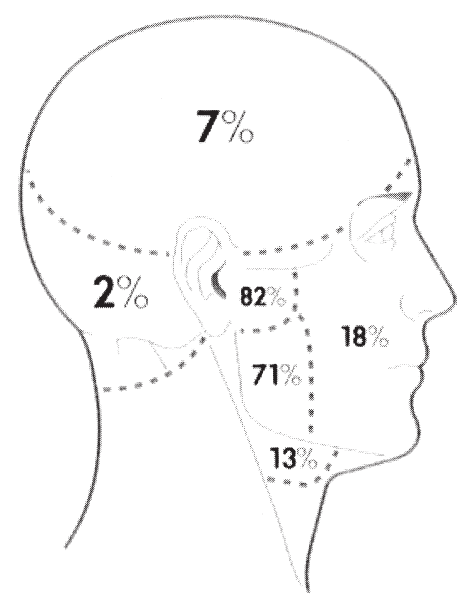

b

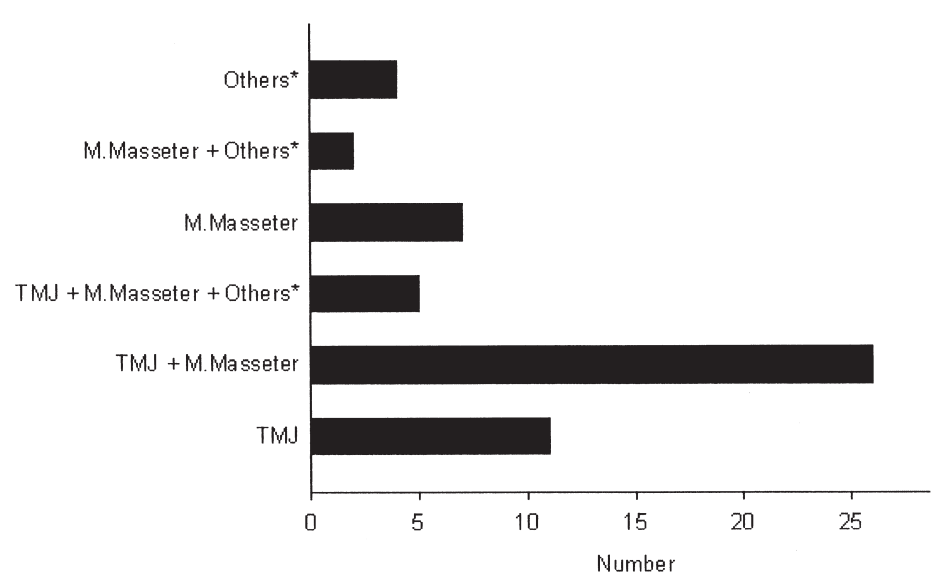

Figure 3. Distribution of orofacial pain. A. Relative distribution of the self-reported orofacial pain localization. Arthritis-related orofacial symptoms were seen in relation to a total of 55 temporomandibular joints (TMJ). B. Distribution of pain localization. * "Others" refers to diffuse orofacial symptoms radiating to different areas such as the infraorbital region, temporal muscle, neck region, or the region of the platysma muscle.

scores for minimal, average, and maximal orofacial pain. The mean scores of minimal and average orofacial pain rose between the first and the second assessment in contrast to the mean scores of maximal pain. A systematic time trend was observed: the mean VAS scores of the variable of average orofacial pain significantly increased between $\mathrm{T} 1$ and T2. Table 3 illustrates the estimated SEM, the confidence levels, and the SDD for each of the variables. Comparable levels of SDD values were seen between minimal, average, and maximal orofacial pain. For a change 
Table 2. Within-patient agreement between the first and the second mean VAS score for minimal, average, and maximal pain shown in the last 3 columns. A significant difference between T1 and T2 scores was seen in the scores of average orofacial pain. For completeness, the average T1 and T2 scores and SD are given in columns 2 and 3 . The large SD are due to a large variation between patients.

\begin{tabular}{lccccc}
\hline Orofacial Pain & $\begin{array}{c}\text { T1 VAS } \\
\text { Score, } \mathrm{mm} \\
(\mathrm{SD})\end{array}$ & $\begin{array}{c}\text { T2 VAS } \\
\text { Score, } \mathrm{mm} \\
(\mathrm{SD})\end{array}$ & $\begin{array}{c}\text { Mean } \\
\text { Difference, mm } \\
(\mathrm{SD})\end{array}$ & $\begin{array}{c}\text { 95\% CL } \\
\text { of Mean } \\
\text { Difference, mm }\end{array}$ & $\begin{array}{c}\text { Significance } \\
\text { T1 vs T2, } \\
\mathrm{p}\end{array}$ \\
\hline $\begin{array}{l}\text { Minimal } \\
\text { Average }\end{array}$ & $17.9(17.1)$ & $18.6(16.3)$ & $0.6(5.2)$ & $-1.2-2.5$ & NS \\
Maximal & $38.4(17.3)$ & $40.9(18.9)$ & $2.4(6.1)$ & $0.3-4.6$ & $<0.05$ \\
& $64.8(16.1)$ & $63.5(16.1)$ & $-1.3(6.9)$ & $-3.7-1.2$ & NS \\
\hline
\end{tabular}

VAS: visual analog scale; CL: confidence level.

Table 3. Values for standard error of the measurement (SEM), confidence level (CL), and smallest detectable difference (SDD) on a visual analog scale. The SDD is the minimal amount of change that can be identified clinically between 2 consecutive observations.

\begin{tabular}{lccc}
\hline Orofacial Pain & SEM, mm & 95\% CL, mm & SDD, mm \\
\hline Minimal & 3.66 & 7.18 & 10 \\
Average & 4.58 & 8.97 & 13 \\
Maximal & 4.89 & 9.6 & 14 \\
\hline
\end{tabular}

between 2 independent observations to be characterized as statistically/clinically significant, it had to at least exceed the SDD of the measurement procedure for the variable in question.

\section{DISCUSSION}

Knowledge about the clinical manifestation of TMJ arthritis-related orofacial symptoms is important for the diagnosis, the choice of treatment, and the evaluation of a therapeutic intervention that aims to relieve these symptoms. The orofacial symptoms presented in our study are generally in keeping with those reported in previous studies of TMJ arthritis in patients with JIA ${ }^{8,11,12}$. In our study, the most frequently reported symptoms were daily pain during mastication, maximal mouth opening, and TMJ morning stiffness. The affected pain locations in our study were more narrowly confined to the TMJ area and the masseter muscle region than in previous studies $8,11,12$. However, any further direct comparison between the findings of previous studies and ours would be misleading because of differences in study objectives and inclusion criteria.

The detailed mechanism behind TMJ arthritis-related pain spread has not been elucidated. However, the orofacial symptoms and limitations in jaw movements seen in patients with TMJ arthritis are comparable to those encountered in other conditions covered by the umbrella term temporomandibular disorders (TMD): in general, TMD are characterized by peripheral and central sensitization as well as a decrease in the activity of the antinociceptive descending pathways. These conditions give rise to phenomena such as allodynia, hyperalgesia, pain spread, referred pain, and a functional musculoskeletal imbalance causing contraction and tension of the masticatory muscles ${ }^{19,20}$. If central sensitization occurs, symptoms can be present without active TMJ inflammation ${ }^{21}$. TMJ arthritis is thus a condition that involves more than the TMJ itself; a small fraction of our patients' complaints were related to myogenic functional symptoms and not to pain from the TMJ area (Figure 3). Our clinical experience indicates that management of TMJ arthritis-related orofacial symptoms is most effectively achieved by a combination of antiinflammatory therapy against TMJ inflammation per se, and interventions that aim to relieve the secondary myogenic functional problem that follows the primary TMJ arthritic condition. We therefore suggest that antiinflammatory actions, such as an increase in general medication and possibly intraarticular steroid injections, be combined with orofacial pain management strategies such as a stabilizing splint, physiotherapy, and general information on painavoidance strategies. Importantly, before initiating a change in the general antiinflammatory medication, one must consider that orofacial symptoms in patients with JIA can originate from a noninflammatory TMD - a condition that is seen in $7 \%$ of an adolescent population, with a higher prevalence of TMD pain among girls than among boys ${ }^{22}$.

One limitation of our study is our inability to conduct routine magnetic resonance imaging (MRI) procedures for all patients in relation to the TMJ arthritis diagnosis, which would have allowed us to evaluate the TMJ arthritis status at the time of inclusion and to exclude patients without current TMJ inflammation. However, this may amount to only a minor weakness because recent research has shown only a limited association between clinical findings and TMJ MRI findings in patients with JIA 7,9,21,23. These findings allow us to suggest that orofacial symptoms are a product of the primary TMJ inflammation in combination with potential secondary myogenic and functional issues that may be at play, as well as phenomena such as allodynia, hyperalgesia, pain spread, and referred pain due to the primary TMJ inflammation. It is also suspected that in the absence of TMJ inflammation, TMJ-erosive osseous changes may lead to Personal non-commercial use only. The Journal of Rheumatology Copyright @ 2012 . All rights reserved. 
suboptimal joint function and pain during function in some patients with JIA $7,9,21,23$. This possibility may explain the orofacial symptoms in patients with JIA who are nonresponders to intraarticular TMJ steroid therapy. This supports a dual treatment approach against TMJ arthritis-related orofacial symptoms in which antiinflammatory actions are combined with other pain management strategies. It is also an argument for an interdisciplinary treatment approach that involves pediatric rheumatologists, specially trained dentists, and physiotherapists.

In our study, the estimated values of the SDD for minimal, average, and maximal orofacial pain were between 10 and $14 \mathrm{~mm}$ on the VAS scale. However, future studies involving more patients are needed to confirm our findings before definitive conclusions on the estimates of SDD can be made. The SDD of patients with JIA in our study were considerably smaller than previously published estimated values for SDD in adult patients with painfully restricted mandibular function ${ }^{24}$. In those patients, Kropmans, et al estimated SDD values for minimal, average, and maximal pain of $25 \mathrm{~mm}, 35 \mathrm{~mm}$, and $43 \mathrm{~mm}$, respectively. These much larger values reflect a considerable variation in the patients' pain perception over time during the 7-day span between the first and second VAS. According to Kropmans, et $a l$, the single largest variance component lay in the interaction between patients and days (variation of pain and time), which produced $45 \%-63 \%$ of the absolute variance between the first and the second $\mathrm{VAS}^{24}$. If we hypothesize that the patients with JIA in our study experience the same inter-day variation, the "true" values of the SDD found in our study may underestimate pain because they do not include the variation in pain over time. In our study, the time between the first and the second VAS was $45 \mathrm{~min}$, which could bring the generalizability of the results into question. However, for ethical reasons, we were unable to postpone treatment initiation. An argument in favor of estimating SDD values on the basis of repeated intra-day VAS scores is that patients' beliefs and perception of pain may fluctuate over time in such a manner that variation in inter-day VAS scores may also reflect additional changes in pain perception and not only the intra-day within-patient variation. It cannot be assumed that the beliefs and perception of TMJ arthritis-related orofacial pain of patients with JIA remain at the same level days apart - especially because our study illustrates large fluctuations in pain frequencies.

Our findings also invite a more general discussion. A significant within-patient difference in average orofacial pain assessment was observed even if assessments were made by the same patients on the same day. We would therefore expect these pain assessments to be comparable and not to differ significantly from each other as seen in Table 2. However, our study reveals that the issue of reliability must be addressed in clinical studies that involve these kinds of assessments. Clinical studies may draw incorrect conclusions based on statistically significant differences between pretherapeutic and posttherapeutic values if an evaluation of the results does not take into consideration the concept of the SDD of the measurement. To demonstrate clinical relevance, clinical intervention must satisfy 2 equally important requirements: (1) a statistically significant difference between the pretherapeutic and posttherapeutic VAS values; and (2) any significant change in the value scores must be comparable to or exceed the SDD of the specific outcome variable examined. One must also consider whether the statistically significant change in the specific measurement value is clinically relevant. It is noteworthy that the estimated values of the minimal clinically relevant differences in self-reported VAS scores in children are very similar to the estimated values of SDD presented in our study. Despite differences in methodology, both methods report values between 10 and $14 \mathrm{~mm}$ on a 100-mm VAS ${ }^{25}$.

\section{ACKNOWLEDGMENT}

The authors thank the staff of the rheumatic team at the Orthodontic Department, Aarhus University, Denmark, for their collaboration in this project. We also thank graphic designer Nikolai Lander for his help with the figures.

\section{REFERENCES}

1. Petty RE, Southwood TR, Manners P, Baum J, Glass DN, Goldenberg $\mathbf{J}$, et al. International League of Associations for Rheumatology classification of juvenile idiopathic arthritis: second revision, Edmonton, 2001. J Rheumatol 2004;31:390-2.

2. Arabshahi B, Cron RQ. Temporomandibular joint arthritis in juvenile idiopathic arthritis: The forgotten joint. Curr Opin Rheumatol 2006;18:490-5.

3. Barriga B, Lewis TM, Law DB. An investigation of the dental occlusion in children with juvenile rheumatoid arthritis. Angle Orthod 1974;44:329-35.

4. Kuseler A, Pedersen TK, Herlin T, Gelineck J. Contrast enhanced magnetic resonance imaging as a method to diagnose early inflammatory changes in the temporomandibular joint in children with juvenile chronic arthritis. J Rheumatol 1998;25:1406-12.

5. Pedersen TK, Jensen JJ, Melsen B, Herlin T. Resorption of the temporomandibular condylar bone according to subtypes of juvenile chronic arthritis. J Rheumatol 2001;28:2109-15.

6. Twilt M, Mobers SM, Arends LR, ten Cate R, Suijlekom-Smit L. Temporomandibular involvement in juvenile idiopathic arthritis. J Rheumatol 2004;31:1418-22.

7. Weiss PF, Arabshahi B, Johnson A, Bilaniuk LT, Zarnow D, Cahill AM, et al. High prevalence of temporomandibular joint arthritis at disease onset in children with juvenile idiopathic arthritis, as detected by magnetic resonance imaging but not by ultrasound. Arthritis Rheum 2008;58:1189-96.

8. Bakke M, Zak M, Jensen BL, Pedersen FK, Kreiborg S. Orofacial pain, jaw function, and temporomandibular disorders in women with a history of juvenile chronic arthritis or persistent juvenile chronic arthritis. Oral Surg Oral Med Oral Pathol Oral Radiol Endod 2001;92:406-14.

9. Pedersen TK, Kuseler A, Gelineck J, Herlin T. A prospective study of magnetic resonance and radiographic imaging in relation to symptoms and clinical findings of the temporomandibular joint in children with juvenile idiopathic arthritis. J Rheumatol 2008;35:1668-75. 
10. Muller L, Kellenberger CJ, Cannizzaro E, Ettlin D, Schraner T, Bolt IB, et al. Early diagnosis of temporomandibular joint involvement in juvenile idiopathic arthritis: A pilot study comparing clinical examination and ultrasound to magnetic resonance imaging. Rheumatology 2009;48:680-5.

11. Engstrom AL, Wanman A, Johansson A, Keshishian P, Forsberg M. Juvenile arthritis and development of symptoms of temporomandibular disorders: A 15-year prospective cohort study. J Orofac Pain 2007;21:120-6.

12. Olson L, Eckerdal O, Hallonsten AL, Helkimo M, Koch G, Gare BA. Craniomandibular function in juvenile chronic arthritis. A clinical and radiographic study. Swed Dent J 1991;15:71-83.

13. Dijkstra PU, de Bont LG, Stegenga B, Boering G. Angle of mouth opening measurement: Reliability of a technique for temporomandibular joint mobility assessment. J Oral Rehabil 1995; 22:263-8.

14. Kropmans TJ, Dijkstra PU, Stegenga B, Stewart R, de Bont LG. Repeated assessment of temporomandibular joint pain: Reasoned decision-making with use of unidimensional and multidimensional pain scales. Clin J Pain 2002;18:107-15.

15. Harris EF, Smith RN. Accounting for measurement error: A critical but often overlooked process. Arch Oral Biol 2009;54 Suppl 1: 107-17.

16. Nilsson IM, List T, Drangsholt M. The reliability and validity of self-reported temporomandibular disorder pain in adolescents. J Orofac Pain 2006;20:138-44.

17. Kropmans TJ, Dijkstra PU, Stegenga B, Stewart R, de Bont LG. Smallest detectable difference in outcome variables related to painful restriction of the temporomandibular joint. J Dent Res 1999;78:784-9.
18. Guyatt G, Walter S, Norman G. Measuring change over time: Assessing the usefulness of evaluative instruments. J Chronic Dis 1987;40:171-8.

19. Cairns BE. Pathophysiology of TMD pain - Basic mechanisms and their implications for pharmacotherapy. J Oral Rehabil 2010;37:391-410.

20. Miles TS, Nauntofte B, Svensson P. Orofacial pain. In: Miles TS, Nauntofte B, Svensson P. Clinical oral physiology. London: Quintessence Publishing; 2004:93-139.

21. Arvidsson LZ, Smith HJ, Flato B, Larheim TA. Temporomandibular joint findings in adults with long-standing juvenile idiopathic arthritis: CT and MR imaging assessment. Radiology 2010;256:191-200.

22. Wahlund K. Temporomandibular disorders in adolescents. Epidemiological and methodological studies and a randomized controlled trial. Swed Dent J 2003;Suppl 164:1-64.

23. Kuseler A, Pedersen TK, Gelineck J, Herlin T. A 2 year followup study of enhanced magnetic resonance imaging and clinical examination of the temporomandibular joint in children with juvenile idiopathic arthritis. J Rheumatol 2005;32:162-9.

24. Kropmans TJ, Dijkstra PU, van Veen A, Stegenga B, de Bont LG. The smallest detectable difference of mandibular function impairment in patients with a painfully restricted temporomandibular joint. J Dent Res 1999;78:1445-9.

25. Powell CV, Kelly AM, Williams A. Determining the minimum clinically significant difference in visual analog pain score for children. Ann Emerg Med 2001;37:28-31. 